\title{
BEM-ESTAR PSICOLÓGICO DA POPULAÇÃO PORTUGUESA
}

\author{
Stefanin Simone Cruz Lima \\ Escola Superior de Saúde, Instituto Politécnico de Bragança. Portugal \\ stefaninlima92@gmail.com \\ Sara Emanuel Afonso Gouveia \\ Enfermeira \\ Eugénia Maria Garcia Jorge Anes \\ Instituto Politécnico de Bragança, Investigadora Integrada da \\ UICISA: e professora na Escola de Saúde IPB. Bragança, Portugal \\ Manuel Alberto Morais Brás \\ Instituto Politécnico de Bragança, Investigador Integrado no \\ CINTESIS e Professor na Escola Superior de Saúde de Bragança, Portugal \\ Maria Fátima Pereira Geraldes \\ Enfermeira, ULSNe. Portugal
}

Recepción Artículo: 17 mayo 2021

Admisión Evaluación: 17 mayo 2021

Informe Evaluador 1: 19 mayo 2021

Informe Evaluador 2: 21 mayo 2021

Aprobación Publicación: 01 junio 2021

\section{RESUMO}

0 bem-estar psicológico pode ser entendido como o equilíbrio emocional entre 0 interno de cada individuo e as vivências externas, ou seja, é estar bem com ele próprio e simultaneamente com os outros, aceitar as exigências e dificuldades da vida, saber lidar, enfrentar ou gerir as emoções, maximizando a plenitude da vida com respeito. A investigação tem vindo a demostrar a enorme importância existente entre 0 bem-estar psicológico e uma boa saúde mental. 0 objetivo do presente estudo é avaliar o nível de bem-estar psicológico na população portuguesa, bem como identificar fatores determinantes. É um estudo de natureza quantitativa, caraterizando-se como observacional, transversal e analítico. Para a seleção da amostra será utilizada a metodologia de Bola de Neve ou Snowball Sampling. Assim, esta denomina-se não probabilística e acidental. Por analogia, é como uma bola de neve que rola monte abaixo e à medida que rola vai aumentando o seu tamanho. Foram respeitados todos os preceitos éticos aplicado a este tipo de investigação, tendo em conta a Declaração de Helsínquia e Convenção de Oviedo. A colheita de dados foi efetuada em 2020. Para tal aplicamos um questionário online constituído por duas partes, a primeira é constituída por questões de caraterização sociodemográfico (género, idade, estado civil, habilitações literárias, naturalidade, distrito, concelho e meio de residência, tipo de habitação), a segunda é constituída pela Escala de Medida de Manifestação de Bem-estar Psicológico (EMMBEP). A amostra é constituída por 135 participantes dos quais 92 são do sexo feminino e 43 do sexo masculino, com idades compreendidas entre os 18 e 63 anos, onde o grupo etário mais frequente é entre os 18 e os 39 anos (73\%) e maioritariamente solteiros $(60,74 \%)$. Em termos profissionais o grupo mais representativo corresponde aos trabalhadores ativos (56,20\%), 


\section{BEM-ESTAR PSICOLÓGICO DA POPULAÇÃO PORTUGUESA}

destes 33,87\% estão a trabalhar em casa e 16,94\% estão a trabalhar on-line. Residem maioritariamente em zona urbana $(70,37 \%$ ) e em moradias (50,37\%). Em termos de saúde 79,26\% referem não ser portadores de doenças e não fazer calmantes/antidepressivos (87,41\%). Relativamente ao bem-estar psicológico, embora sem significância estatística, parecem verificar-se valores médios superiores no sexo masculino $(70,93)$, nos indivíduos com 60 e mais anos $(69,66)$, nos divorciados $(73,66)$, nos estudantes $(70)$, nos trabalhadores a exercer a atividade no local de trabalho $(69,04)$, nos indivíduos a residir em zona urbana $(68,57)$ e em apartamentos $(68,61 \%)$, nos participantes que afirmam não ser doentes $(69,21)$. E naqueles que tomam calmantes/antidepressivos $(72,5)$. Os trabalhadores em Lay-off (46) e os reformados (41) apresentam em média, scores abaixo de 60. Em conclusão, observamos valores superiores a 60 para grande maioria da amostra, apresentando em geral bons níveis de bemestar psicológico. Com exceção dos trabalhadores em Lay-off e dos reformados. Não foram identificadas relações estatísticas com os fatores analisados. Estes dados devem ser tidos em conta no que respeita à tomada de decisão em saúde, uma vez que são identificados grupos com baixo bem-estar psicológico, onde a intervenção poderá ter importância fulcral, contribuindo para elevar o nível de bem-estar e por conseguinte 0 estado de saúde das populações. 0 tamanho da amostra poderá ser considerado limite à presente investigação

Palavras-chave: bem-estar psicológico; esscala de medida de manifestação de bem-estar psicológico.

\section{ABSTRACT}

Psychological well-being of the portuguese population. Psychological well-being can be understood as the emotional balance between the internal of each individual and the external experiences, that is, it means being well with oneself and simultaneously with others, accepting the demands and difficulties of life, knowing how to deal, cope or manage emotions, maximizing the fullness of life with respect. Research has been showing the enormous importance between psychological well-being and good mental health. The aim of the present study is to assess the level of psychological well-being in the Portuguese population, as well as to identify determining factors. It is a quantitative study, characterized as observational, transversal and analytical. For the sample selection, the Snowball or Snowball Sampling methodology was used. Thus, this is called non-probabilistic and accidental. By analogy, it is like a snowball that rolls down the hill and as it rolls it increases in size. All ethical principles applied to this type of research were respected, considering the Declaration of Helsinki and the Oviedo Convention. Data collection was carried out in 2020. To this end, we applied an online questionnaire consisting of two parts, the first of which consists of questions of a sociodemographic character (gender, age, marital status, educational qualifications, place of birth, district, municipality and means of residence, type of housing), the second is made up of the Psychological Well-Being Manifestation Scale (EMMBEP). The sample consists of 135 participants, of which 92 are female and 43 are male, aged between 18 and 63 years, where the most frequent age group is between 18 and 39 years (73\%) and mostly single (60.74\%). In professional terms, the most representative group corresponds to active workers (56.20\%), of these $33.87 \%$ are working at home and $16.94 \%$ are working online. They mostly live in urban areas (70.37\%) and in houses (50.37\%). In terms of health, $79.26 \%$ reported not having diseases and not taking tranquilizers / antidepressants (87.41\%). Regarding psychological well-being, although without statistical significance, higher mean values seem to be observed in males (70.93), in individuals aged 60 and over (69.66), in divorced people (73.66), in students (70), workers working in the workplace (69.04), individuals living in urban areas (68.57) and apartments (68.61\%), participants who say they are not sick (69.21) and who take tranquilizers / antidepressants (72.5). Lay-off workers (46) and pensioners (41) have, on average, scores below 60 . In conclusion, we observed values above 60 for most people in the sample, with generally good levels of psychological well-being. Except for workers in Lay-off and pensioners. No statistical relationship was identified with the factors analyzed. These data must be considered regarding health decision-making, since groups with low psychological well-being are identified, where the intervention may be of central importance, contributing to raise the level of well-being and therefore the health status of the populations. The sample size may be considered a limit to the present investigation

Keywords: psychological well-being; psychological sell-being manifestation measure scale 


\section{INTRODUÇÃO}

0 estudo do Bem-estar começou a despertar interesse na Grécia antiga com os filósofos Aristóteles e Aristipo, na tentativa de decifrarem em que consistia uma existência feliz e o bem-viver. Em 1960 que os investigadores começaram a ter evidências científicas sobre 0 tema, introduzindo 0 conceito no âmbito da psicologia, tornando-0 um dos temas mais discutidos (Siqueira \& Padovam, 2008). Com a segunda guerra mundial houve uma necessidade de avaliar questões sobre a doença mental, doença e sofrimento humano e pouco explorados aspetos como a saúde mental e felicidade, o que acabou por tornar o bem-estar um investimento de domínio da psicologia positiva. (Wagner de Lara Machado, Denise Ruschel Bandeira, 2012).

0 conceito de bem-estar, provem da discussão filosófica e científica entre hedonismo (prazer; vontade) e eudaimonia (felicidade), que deram origem aos dois modelos de bem-estar. No contexto da filosofia aristotélica a BEP, o termo eudaimonia tem sido utilizado como sinonimo de felicidade como sendo a principal motivação da existência humana, reconhecendo assim a necessidade da pessoa viver de acordo com o seu daimon ou self, isto é, de acordo com as suas máximas capacidades, de forma a conseguir a sua realização pessoal (Novo, 2003; Ryff \& Keyes, 1995).

0 bem-estar psicológico pode ser entendido como o equilíbrio emocional entre 0 interno de cada individuo e as vivências externas, ou seja, é estar bem com ele próprio e simultaneamente com os outros, aceitar as exigências e dificuldades da vida, saber lidar, enfrentar ou gerir as emoções, maximizando a plenitude da vida com respeito. A investigação tem vindo a demostrar a enorme importância existente entre 0 bem-estar psicológico e uma boa saúde mental.

\section{OBJETIVO}

0 objetivo do presente estudo é avaliar o nível de bem-estar psicológico na população portuguesa, bem como identificar fatores determinantes.

\section{METODOLOGIA}

É um estudo de natureza quantitativa, caraterizando-se como observacional, transversal e analítico. Para a seleção da amostra será utilizada a metodologia de Bola de Neve ou Snowball Sampling. Assim, esta denominase não probabilística e acidental. Por analogia, é como uma bola de neve que rola monte abaixo e à medida que rola vai aumentando o seu tamanho. Foram respeitados todos os preceitos éticos aplicado a este tipo de investigação, tendo em conta a Declaração de Helsínquia e Convenção de Oviedo. A colheita de dados foi efetuada em 2020. Para tal aplicamos um questionário online constituído por duas partes, a primeira é constituída por questões de caraterização sociodemográfico (género, idade, estado civil, habilitações literárias, naturalidade, distrito, concelho e meio de residência, tipo de habitação), a segunda é constituída pela Escala de Medida de Manifestação de Bem-estar Psicológico (EMMBEP). É uma escala de resposta tipo likert de 5 pontos, que vão desde 1 (Nunca) a 5 (Quase sempre), constituída por um total de 25 questões, dividida em seis subescalas: autoestima (4 itens), equilíbrio (4 itens), envolvimento social (4 itens), sociabilidade (4 itens), controlo de si e dos acontecimentos (4 itens) e felicidade (5 itens). Quanto mais elevado for o total obtido - dado pela soma das pontuações de todos os itens - maior será o bem-estar psicológico percebido. Para inclusão no estudo considerou-se toda a população residente em Portugal, com mais de 18 anos de idade e aceitassem participar no estudo.

\section{RESULTADOS}

A amostra (Quadro 1) é constituída por 135 participantes dos quais 92 são do sexo feminino e 43 do sexo masculino, com idades compreendidas entre os 18 e 63 anos, onde o grupo etário mais frequente é entre os 18 e os 39 anos (73\%) e maioritariamente solteiros $(60,74 \%)$. Em termos profissionais o grupo mais representativo corresponde aos trabalhadores ativos (56,20\%), destes 33,87\% estão a trabalhar em casa e 16,94\% estão a trabalhar on-line. Residem maioritariamente em zona urbana (70,37\%) e em moradias (50,37\%). 
BEM-ESTAR PSICOLÓGICO DA POPULAÇÃO PORTUGUESA

Quadro 1 - Caraterização sociodemográfica e laboral da amostra.

\begin{tabular}{|c|c|c|c|c|c|c|c|c|c|}
\hline Sexo & $\bar{n}$ & \multicolumn{2}{|c|}{$\overline{\%}$} & & & & \multirow{2}{*}{$\begin{array}{l}\text { Meio de } \\
\text { Residênc } \\
\text { ia }\end{array}$} & \multirow{2}{*}{ n } & \multirow{2}{*}{$\%$} \\
\hline Feminino & 92 & \multicolumn{2}{|c|}{68,15} & Estado Civil & n & $\%$ & & & \\
\hline Masculin & 43 & \multicolumn{2}{|c|}{31,85} & Solteiro/a & 82 & 60,74 & \multirow[b]{2}{*}{ Urbano } & \multirow[b]{2}{*}{95} & \multirow[b]{2}{*}{70,3} \\
\hline Total & 135 & \multicolumn{2}{|c|}{100,00} & $1 \mathrm{em}$ & & & & & \\
\hline & & \multirow{2}{*}{\multicolumn{2}{|c|}{$\%$}} & União de facto & 48 & 35,56 & Rural & 40 & 29,63 \\
\hline Idade & n & & & Divorciado/a ou & & & Total & 135 & 100 \\
\hline 18-39 & 99 & \multicolumn{2}{|c|}{73,00} & Separado/a & 3 & 2,22 & \multirow{2}{*}{$\begin{array}{l}\text { Tipo de } \\
\text { habitação }\end{array}$} & \multirow[b]{2}{*}{ n } & \multirow[b]{2}{*}{$\%$} \\
\hline $40-59$ & 33 & \multicolumn{2}{|c|}{24,00} & Viúvo/a & 2 & 1,48 & & & \\
\hline $\begin{array}{l}60 \text { ou } \\
\text { mais }\end{array}$ & 3 & \multicolumn{2}{|c|}{2,00} & Total & $\begin{array}{l}13 \\
5\end{array}$ & 100 & \multicolumn{2}{|c|}{$\begin{array}{l}\text { Apartament } \\
\text { o }\end{array} 67$} & $\begin{array}{l}49,6 \\
3\end{array}$ \\
\hline \multirow[t]{2}{*}{ Total } & \multirow[t]{2}{*}{135} & \multirow{2}{*}{\multicolumn{2}{|c|}{100}} & & & & & 68 & $\begin{array}{l}50,3 \\
7\end{array}$ \\
\hline & & & & & & & \multirow{2}{*}{$\begin{array}{l}\text { Moradia } \\
\text { Total } \\
\end{array}$} & 13 & 5100 \\
\hline \multicolumn{2}{|l|}{ Ocupação } & $\bar{n}$ & $\%$ & \multicolumn{3}{|c|}{ Situação atual de trabalho } & & $\bar{n}$ & $\%$ \\
\hline \multicolumn{2}{|l|}{ Estudante } & 47 & 34,81 & \multicolumn{4}{|c|}{ Não trabalho, estou em casa } & 58 & 46,77 \\
\hline \multicolumn{2}{|c|}{$\begin{array}{l}\text { Trabalhador } \\
\text { estudante }\end{array}$} & 14 & 10,37 & \multicolumn{4}{|c|}{$\begin{array}{l}\text { Sim, estou a trabalhar no meu local de } \\
\text { trabalho }\end{array}$} & 42 & 33,87 \\
\hline \multicolumn{2}{|c|}{ Trabalhador } & 62 & 45,93 & \multirow{2}{*}{\multicolumn{4}{|c|}{ Sim, estou a trabalhar a partir de casa }} & & \\
\hline \multirow{2}{*}{\multicolumn{2}{|c|}{$\begin{array}{l}\text { Desempregad } \\
0\end{array}$}} & & & & & & & 21 & 16,94 \\
\hline & & 10 & 7,41 & \multicolumn{4}{|l|}{ Sim em Lay-off } & 2 & 2,42 \\
\hline \multicolumn{2}{|c|}{ Reformado } & 2 & 1,48 & Total & & & & 124 & 100 \\
\hline Total & & 135 & 100 & & & & & & \\
\hline
\end{tabular}

Em termos de saúde (Quadro 2) 79,26\% referem não ser portadores de doenças e não fazer calmantes/antidepressivos $(87,41 \%)$.

Quadro 2 - Caraterização de saúde da amostra

\begin{tabular}{|l|l|l|l|l|l|}
\hline Doença Crónica & \multicolumn{1}{|c|}{$\mathbf{n}$} & $\mathbf{\%}$ & Calmantes & $\mathbf{n}$ & $\mathbf{\%}$ \\
\hline Não & 107 & 79,26 & Não toma & 11887,41 \\
\hline Sim & 28 & 20,74 & Toma & 17 & 12,59 \\
\hline Total & 135 & 100 & Total & 135100 \\
\hline
\end{tabular}

Relativamente ao bem-estar psicológico, embora sem significância estatística, parecem verificar-se valores médios superiores no sexo masculino $(70,93)$, nos indivíduos com 60 e mais anos $(69,66)$, nos divorciados $(73,66)$, nos estudantes (70), nos trabalhadores a exercer a atividade no local de trabalho $(69,04)$, nos indivíduos a residir em zona urbana $(68,57)$ e em apartamentos $(68,61 \%)$, nos participantes que afirmam não ter doenças crónicas $(69,21)$ e naqueles que tomam calmantes/antidepressivos $(72,5)$. 
Quadro 3 - Bem Estar Psicológico EMMBEP: relação com as variáveis de caraterização.

\begin{tabular}{|c|c|c|c|c|}
\hline \multicolumn{2}{|r|}{ Variáveis } & Média & DP & $P$ \\
\hline \multirow{2}{*}{ Género } & Feminino & 66,63 & 17,07 & \multirow{2}{*}{0,63} \\
\hline & Masculino & 70,93 & 5920 & \\
\hline \multirow{3}{*}{ Idade } & $18-39$ & 68,89 & 18,38 & \multirow{3}{*}{0,532} \\
\hline & $40-59$ & 65,15 & 18,18 & \\
\hline & $60+$ anos & 69,66 & 20,03 & \\
\hline \multirow{4}{*}{$\begin{array}{c}\text { Estado } \\
\text { civil }\end{array}$} & Solteiro & 67,68 & 18,16 & \multirow{4}{*}{0,803} \\
\hline & Casado & 68,43 & 19,26 & \\
\hline & Divorciado & 73,66 & 15,82 & \\
\hline & Viuvo & 62 & 4,24 & \\
\hline \multirow{5}{*}{ Ocupação } & Estudante & 70 & 18,62 & \multirow{5}{*}{0,155} \\
\hline & Trab/estud & 62,28 & 16,19 & \\
\hline & Trabalhador & 69,42 & 18,45 & \\
\hline & Desempregado & 60,9 & 17,35 & \\
\hline & Reformado & 52,5 & 16,26 & \\
\hline \multirow{4}{*}{$\begin{array}{l}\text { Situação } \\
\text { atual }\end{array}$} & Não trabalho & 67,27 & 17,35 & \multirow{4}{*}{0,823} \\
\hline & $\begin{array}{l}\text { Trabalho no local } \\
\text { de trabalho }\end{array}$ & 69,04 & 16,86 & \\
\hline & $\begin{array}{l}\text { Trabalho a partir de } \\
\text { casa }\end{array}$ & 68 & 22,01 & \\
\hline & Lay-off & 78,5 & 20,5 & \\
\hline \multirow{2}{*}{$\begin{array}{l}\text { Meio de } \\
\text { residencia }\end{array}$} & Urbano & 68,57 & 18,36 & \multirow{2}{*}{0,391} \\
\hline & Rural & 66,62 & 18,28 & \\
\hline \multirow{2}{*}{$\begin{array}{c}\text { Tipo de } \\
\text { habitação }\end{array}$} & Apartamento & 68,61 & 18,55 & \multirow{2}{*}{0,563} \\
\hline & Moradia & 67,39 & 18,16 & \\
\hline \multirow{2}{*}{$\begin{array}{l}\text { Doença } \\
\text { Crónica }\end{array}$} & Não & 69,21 & 18,43 & \multirow{2}{*}{0,105} \\
\hline & Sim & 63,35 & 17,3 & \\
\hline \multirow{2}{*}{$\begin{array}{l}\text { Consumo } \\
\text { Calmantes } \\
\end{array}$} & Sim & 70,53 & 17,2 & \multirow{2}{*}{0,681} \\
\hline & Não & 67,63 & 18,49 & \\
\hline
\end{tabular}

Os trabalhadores em Lay-off (46) e os reformados (41) apresentam em média, scores abaixo de 60 para 0 bem-estar psicológico.

\section{CONCLUSÕES}

Em conclusão, embora não tenham sido identificadas relações estatísticas com os fatores analisados, observamos valores superiores a 60 para grande maioria da amostra, apresentando em geral bons níveis de bem-estar psicológico. Com exceção dos trabalhadores em Lay-off e dos reformados. Não. Estes dados devem ser tidos em conta no que respeita à tomada de decisão em saúde, uma vez que são identificados grupos com baixo bem-estar psicológico, onde a intervenção poderá ter importância fulcral, contribuindo para elevar o nível de bem-estar e 


\section{BEM-ESTAR PSICOLÓGICO DA POPULAÇÃO PORTUGUESA}

por conseguinte 0 estado de saúde das populações. 0 tamanho da amostra poderá ser considerado limite à presente investigação. Estas avaliações permitem avaliar o nível de bem-estar das populações e a sua relação com os seus determinantes. Indispensáveis à tomada de decisão política e de saúde.

\section{REFERÊNCIAS BIBLIOGRÁFICAS}

Novo, R. (2003). Para além da eudaimonia: 0 bem-estar psicológico em mulheres na idade adulta avançada. Fundação Calouste Gulbenkian / Fundação para a Ciência e a Tecnologia / Ministério da Ciência e do Ensino Superior. Coimbra: Dinalivro.

Ryff, C. D., \& Keyes, C. L. M. (1995). The structure of psychological well-being revisited. Journal of Personality and Social Psychology, 69(4), 719-727. https://doi.org/10.1037/0022-3514.69.4.719

Siqueira, M. M. M., \& Padovam, V. A. R. (2008). Bases teóricas de bem-estar subjetivo, bem-estar psicólogico e bem-estar no trabalho [Theoretical basis of subjective well-being, psychological well-being and well-being at work]. Psicologia: Teoria e Pesquisa, 24(2), 201-209. https://doi.org/10.1590/S010237722008000200010

Wagner de Lara Machado, Denise Ruschel Bandeira. (2012). Bem-estar psicológico: definição, avaliação e principais correlatos. Em D. R. Wagner de Lara Machado, \& Machado, Bandeira, Estudos de psicologia (pp. 587595). Campinas. 